\title{
Caracterização sociodemográfica e clínica dos pacientes hipertensos não controlados atendidos em uma unidade de pronto atendimento
}

RESUMO|A hipertensão arterial sistêmica é uma doença crônica não transmissível, considerada um grande problema de saúde pública. Este estudo teve como objetivo caracterizar os hipertensos não controlados atendidos em uma unidade de pronto atendimento. Tratase de um estudo descritivo, transversal e quantitativo. Participaram 122 hipertensos com pressão arterial $\geq 140 \times 90 \mathrm{mmHg}$, durante junho e julho de 2017. Os resultados mostram a predominância de idosos $56,6 \%$ com média de $61,25( \pm 14,48)$ anos, sexo feminino $65,6 \%$, brancos $59 \%$, casados $49,2 \%$, aposentados $45,1 \%$, baixa renda $1,1(0,92)$ e baixa escolaridade $6,35( \pm 5,41)$. Os sujeitos apresentaram nível médio pressórico sistólico $169,3( \pm 20,24) \mathrm{mmHg}$ e diastólico $97,53( \pm 16,49) \mathrm{mmHg}$. Apresentaram Diabetes Mellitus associado, sedentarismo e cefaleia como queixa principal. Estes resultados contribuem para o fomento de outras pesquisas, planejamento, execução e avaliação de medidas de promoção e educação em saúde a fim de garantir o fortalecimento da adesão ao tratamento farmacológico e controle da pressão arterial.

Palavras-chaves: Emergências; Enfermagem; Hipertensão.

ABSTRACT | Systemic arterial hypertension is a non-communicable chronic disease considered a major public health problem. The objective of this study was to characterize the uncontrolled hypertensive patients treated in a care unit. It is a descriptive, cross-sectional and quantitative study. A total of 122 hypertensive individuals with blood pressure $\geq 140 \times 90 \mathrm{mmHg}$ were observed during June and July 2017. The results show a predominance of $56.6 \%$ with a mean of $61.25( \pm 14.48)$ years, female $65.6 \%$, white $59 \%$, married $49.2 \%$, retired $45.1 \%$, low income $1.1(0.92)$ and low education $6.35( \pm 5.41)$. The subjects had mean systolic blood pressure level $169.3( \pm 20.24) \mathrm{mmHg}$ and diastolic blood pressure $97.53( \pm 16.49) \mathrm{mmHg}$. They presented associated Diabetes Mellitus, sedentary lifestyle and headache as the main complaint. These results contribute to the promotion of further research, planning, implementation and evaluation of health promotion and education measures in order to strengthen the adherence to pharmacological treatment and blood pressure control.

Keywords: Emergencies; Hypertension; Nursing.

RESUMEN | La hipertensión arterial sistémica es una enfermedad crónica no transmisible, considerada un gran problema de salud pública. Este estudio tuvo como objetivo caracterizar los hipertensos no controlados atendidos en una unidad de pronta atención. Se trata de un estudio descriptivo, transversal y cuantitativo. Los resultados mostraron la predominancia de los ancianos $56,6 \%$ con una media de $61,25( \pm 14,48)$ años, sexo femenino $65,6 \%$, blancos $59,6 \%$, blancos $59,6 \%$, con una presión arterial $\geq 140 \times 90 \mathrm{mmHg}$, durante junio y julio de 2017 . $\%$, casados $49,2 \%$, jubilados $45,1 \%$, baja renta $1,1(0,92)$ y baja escolaridad $6,35( \pm 5,41)$. Los sujetos presentaron nivel medio presórico sistólico $169,3( \pm 20,24) \mathrm{mmHg}$ y diastólica $97,53( \pm 16,49) \mathrm{mmHg}$. Presentaron la diabetes Mellitus asociada, sedentarismo y cefalea como queja principal. Estos resultados contribuyen al fomento de otras investigaciones, planificación, ejecución y evaluación de medidas de promoción y educación en salud a fin de garantizar el fortalecimiento de la adhesión al tratamiento farmacológico y control de la presión arterial.

Descriptores: Enfermería; Hipertensión; Urgencias-Médicas.

\section{Larissa dos Santos Sousa}

Enfermeira. Residente em Unidade de Terapia Intensiva- SES-PE (COREMU-IMIP), Brasil.

\section{Marina Saraiva de Araújo Pessoa}

Enfermeira. Residente em Saúde da Criança pela SES-PB, Brasil.

\section{Roberta Paolli de Paiva Oliveira}

Enfermeira. Residente em Cardiologia pela UFRN, Brasil.

\section{Aísha Sthéfany Silva de Meneses}

Enfermeira. Residente em Saúde da Família. Programa Multiprofissional em Saúde da Família e da Comunidade pela SMS-JP, Brasil.

\section{Luciene Maria da Costa}

Acadêmica de Enfermagem. Faculdade Maurício de Nassau, Brasil.

\section{Nemório Rodrigues Alves}

Enfermeiro. Residente em Saúde da Família pela UNCISAL, Brasil.

\section{Taciana da Costa Farias Almeida}

Enfermeira. Mestre. Doutoranda em Enfermagem Universidade Federal da Paraíba. Docente do Curso de Enfermagem da Universidade Federal de Campina Grande, Brasil.

Recebido em: 14/03/2019

Aprovado em: 20/05/2019 
INTRODUÇÃO

hipertensão arterial sistê-
mica (HAS) é uma doença
cardiovascular de caráter crônico-degenerativa caracterizada pela elevação sustentada dos níveis pressóricos de pressão arterial sistólica $\geq 140$ e diastólica $\geq 90 \mathrm{mmHg} 1$. No Brasil a HAS é considerada um grave problema de saúde pública que acomete cerca de 31,3 milhões de pessoas2-3.

Os elementos que contribuem para a multideterminação da HAS dividem-se em fatores modificáveis, tais como: estilo de vida (tabagismo, obesidade, etilismo, sedentarismo, estresse, ingestão de sal), escolaridade e fatores socioeconômicos, e fatores não modificáveis, entre eles os aspectos genéticos, que envolvem idade, sexo e história familiar. O processo de envelhecimento é um fator importante para existência de alterações orgânicas, aumentando a pressão sanguínea arterial devido ao acúmulo de placa aterosclerótica, aumento do deposito de colágeno, fragmentação das elastinas arteriais e vasodilatação prejudicada4.

Os pacientes diagnosticados com HAS necessitam de um tratamento adequado e contínuo, entretanto, o descontrole dos níveis pressóricos relacionados a falta de adesão à terapia farmacológica e não farmacológica levam esses indivíduos a procurarem os serviços de urgência e emergência, entre eles, as Unidades de Pronto Atendimento (UPA) 5-6.

Os danos advindos da HAS, como Acidente Vascular Encefálico (AVE), Infarto Agudo do Miocárdio (IAM), Insuficiência Cardíaca (IC), Doença Arterial Periférica (DAP) e Doença Renal Crônica (DRC) prejudicam a qualidade de vida do indivíduo, além de acarretar custos exorbitantes ao Sistema Único de Saúde (SUS) com o tratamento e a reabilitação1,7.

Um estudo realizado na região sudeste do Brasil identificou o perfil dos pacientes hipertensos, admitidos nos setores de emergência, como pertencentes ao sexo feminino, com idade média de 61,5 anos, de baixa escolaridade, seden- tários e com comorbidades, dentre elas a Diabetes Mellitus (DM) e a dislipidemia8.

Com base nesse cenário, este estudo é pioneiro no local de pesquisa elencado. Portanto, pergunta-se: qual o perfil clínico e sociodemográfico dos pacientes hipertensos não controlados atendidos em uma unidade de pronto atendimento? Assim, este estudo objetivou caracterizar os aspectos clínicos e sociodemográficos de pacientes hipertensos não controlados atendidos na UPA $24 \mathrm{~h}$ de um município do interior da Paraíba/BR.

\section{MÉTODO}

Trata-se de um estudo quantitativo, descritivo e de corte transversal, desenvolvido em uma Unidade de Pronto Atendimento (UPA) 24h no município de Campina Grande, no estado da Paraíba, durante o período de junho a juIho de 2017. A escolha da Unidade de Pronto Atendimento se deu por ser uma unidade que é referência inicial para casos de urgências hipertensivas dentro da rede de urgências e emergências do município de Campina Grande e região.

Constituíram a amostra cento e vinte dois pacientes hipertensos com valores pressóricos de $\mathrm{PA} \geq 140 / 90$ (não controlados) que deram entrada na sala de triagem da UPA $24 \mathrm{~h}$ e que se enquadravam nos seguintes critérios: diagnóstico prévio de Hipertensão Arterial Sistêmica; admitidos na unidade de pronto atendimento com $\mathrm{PA} \geq 140 \times 90 \mathrm{mmHg}$; maiores de 18 anos; em uso de medicação para controle de PA; ambos os sexos e com circunferência braquial do braço direito de $22-32 \mathrm{~cm}$. Adotaram-se como critérios de exclusão: portadores de condições clínicas que não possibilitasse responder ao formulário; dependentes de cuidadores para tomar medicamentos; pacientes com transtornos mentais.

Todos os pacientes abordados e que se enquadravam nos critérios de inclusão consentiram em participar do estudo, após assinatura do Termo de Consentimento Livre e Esclarecido (TCLE).
Os dados foram obtidos através de entrevista com um formulário próprio durante o atendimento do cliente na sala de triagem. Esse instrumento foi elaborado com base na literatura e na experiência clínica dos pesquisadores. As variáveis biossociais incluíram: idade, sexo, etnia (cor autodeclarada), se mora acompanhado, estado civil (casado, solteiro, separado e viúvo), ocupação (aposentado, do lar, autônomo, empregado e afastado), escolaridade (anos de estudo) e renda salarial média. Avaliaram-se, ainda, fatores de risco (sedentarismo, tabagismo e alcoolismo). Foram considerados sedentários os indivíduos que não realizavam atividade física por pelo menos três vezes na semana, de acordo com as VII Diretrizes Brasileiras de Hipertensão1, tabagistas aqueles que usavam cigarro, independente do tempo ou quantidade, e alcoolistas àqueles que consumiam $30 \mathrm{~g}$ por dia. Comorbidades referidas como Diabetes Mellitus (DM), dislipidemia, IAM e AVE também foram levantadas. Os dados clínicos coletados foram: queixa relacionada à pressão, tempo de diagnóstico (meses), pressão arterial sistólica (PAS), pressão arterial diastólica (PAD), frequência cardíaca (FC) e circunferência braquial (CB).

Para confirmar o valor da PA e enquadrar os sujeitos na pesquisa, com $\mathrm{PA} \geq 140 \times 90 \mathrm{mmHg}$, as pesquisadoras, após a abordagem inicial das profissionais na sala de triagem, realizavam nova medida da PA. Para este procedimento, foi utilizada fita métrica inelástica para verificar a CB e adequar aqueles sujeitos ao manguito disponível 22-32 cm. As medidas da PA foram realizadas conforme as etapas preconizadas pelas VII Diretrizes Brasileiras de Hipertensão Arterial1 com esfigmomanômetro automático validado, da marca Onrom, modelo Hem-7200. Este equipamento, além da medida da PA, disponibiliza a FC.

Após a coleta, os dados foram inseridos em planilha e posteriormente submetidos à análise estatística e inferencial 
(testes de Mann Whitney e Qui-quadrado de Pearson) por meio do programa estatístico SPSS versão 21.0, e os resultados apresentados de forma descritiva e em tabelas.

A pesquisa foi autorizada pelo Comitê de Ética e Pesquisa do Hospital Universitário Alcides Carneiro, sob parecer n. ${ }^{\circ}$ 2.065.089, CAAE: 65972817.5.0000.5182, e realizada em conformidade com os pressupostos éticos da Resolução no 466/12 do ConseIho Nacional de Saúde - CNS/MS.

\section{RESULTADOS}

Os resultados representam dados socioeconômicos e clínicos dos hipertensos não controlados $(\mathrm{PA} \geq 140 \times 90 \mathrm{mmHg})$ que foram admitidos na sala de triagem da UPA 24h no período de junho a julho de 2017. Para alcançar o maior número de sujeitos, foram realizadas visitas durante 30 dias ao local da pesquisa. No intuito de conhecer e elencar a maior parte da população hipertensa descontrolada, as visitas foram realizadas nos três turnos do dia, sendo abordados 44 sujeitos no turno da manhã, 57 à tarde e 21 à noite.
A amostra foi constituída por 122 sujeitos, destes 80 (65,6\%) eram do sexo feminino. A média de idade dos sujeitos foi de 61,25 $( \pm 14,48)$ anos. Quanto a cor da pele, 72 (59\%) se autodeclararam brancos, $99(81,1 \%)$ dos usuários moravam acompanhados, 60 $(49,2 \%)$ eram casados e apresentavam escolaridade média de $6,35 \quad( \pm 5,42)$ anos de estudos. A ocupação de aposentado prevaleceu $55(45,1 \%)$ e a média da renda familiar foi de 1,1 $( \pm 0,93)$ salários mínimos, conforme observa-se os dados (Tabela 1).

Tabela 1: Caracterização sociodemográfica dos pacientes hipertensos não controlados atendidos na UPA 24h. Campina Grande,

Paraíba, Brasil, 2017. ( $n=122)$

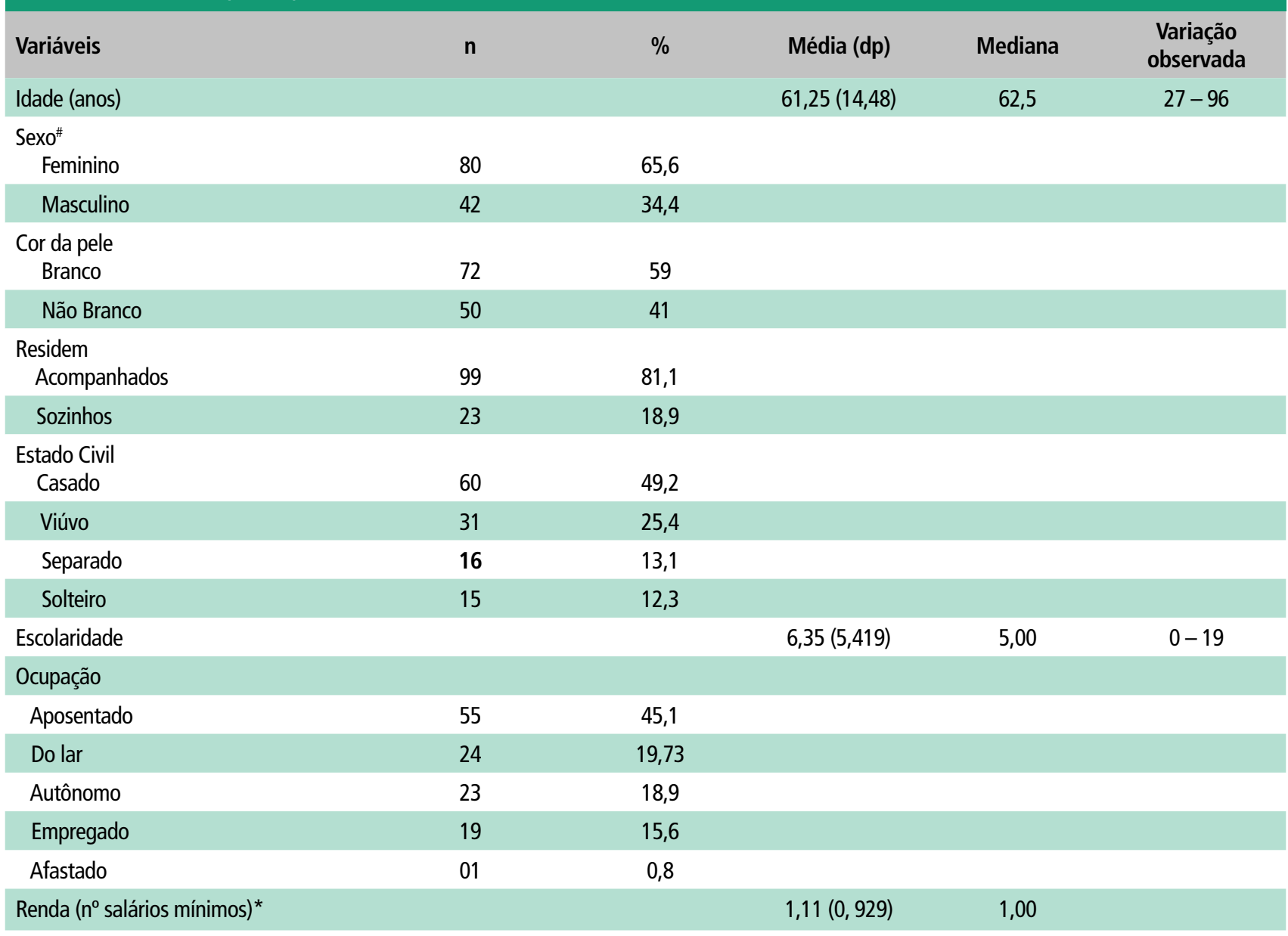

*Salário Mínimo $2017=$ R\$ 937,00

"Teste de Mann Whitney $(\mathrm{p}=0,706)$

Fonte: Dados da Pesquisa. 
De acordo com o teste de Mann Whitney, há evidências estatísticas de que os grupos feminino e masculino apresentam idades medianas iguais $(p=0,706)$.

A média do tempo de diagnóstico de HAS foi de $123,38( \pm 93,60)$ meses - aproximadamente 10 anos, a média da PAS foi de $169,3( \pm 20,24) \mathrm{mmHg}$ e a de PAD foi de $97,53( \pm 16,49) \mathrm{mmHg}$. Quanto a fre- quência cardíaca houve uma média 86,56 $( \pm 17,39)$ bpm e a circunferência braquial média 27,87 $( \pm 2,35) \mathrm{cm}$ (Tabela 2).

A respeito da caracterização clínica, verificou-se que $20(16,4 \%)$ declararam alcoolismo, 29 (23,8\%) tabagismo e 69 (56.6\%) eram sedentários. Não há evidência estatística, de acordo com o teste Qui-quadrado, de que a composição do sexo dos pacientes por fatores de risco para DCV, como sedentarismo $(\mathrm{p}=0,149)$, alcoolismo $(p=0,340)$ e tabagismo $(p=0,367)$ sejam diferentes.

Dentre as morbidades associadas destacou-se a DM apresentada por 37 (30,3\%) sujeitos. Quanto as principais queixas relatadas na busca pelo atendimento na UPA, constatou-se que $52(42,6 \%)$ procuraram pela elevação dos níveis pressóricos e em decorrência de episódios de cefaleia 26 (21,3\%).

Tabela 2: Caracterização clínica dos hipertensos não controlados admitidos na sala de triagem da UPA 24h. Campina Grande, Paraíba, Brasil, 2017. $(\mathrm{n}=122)$

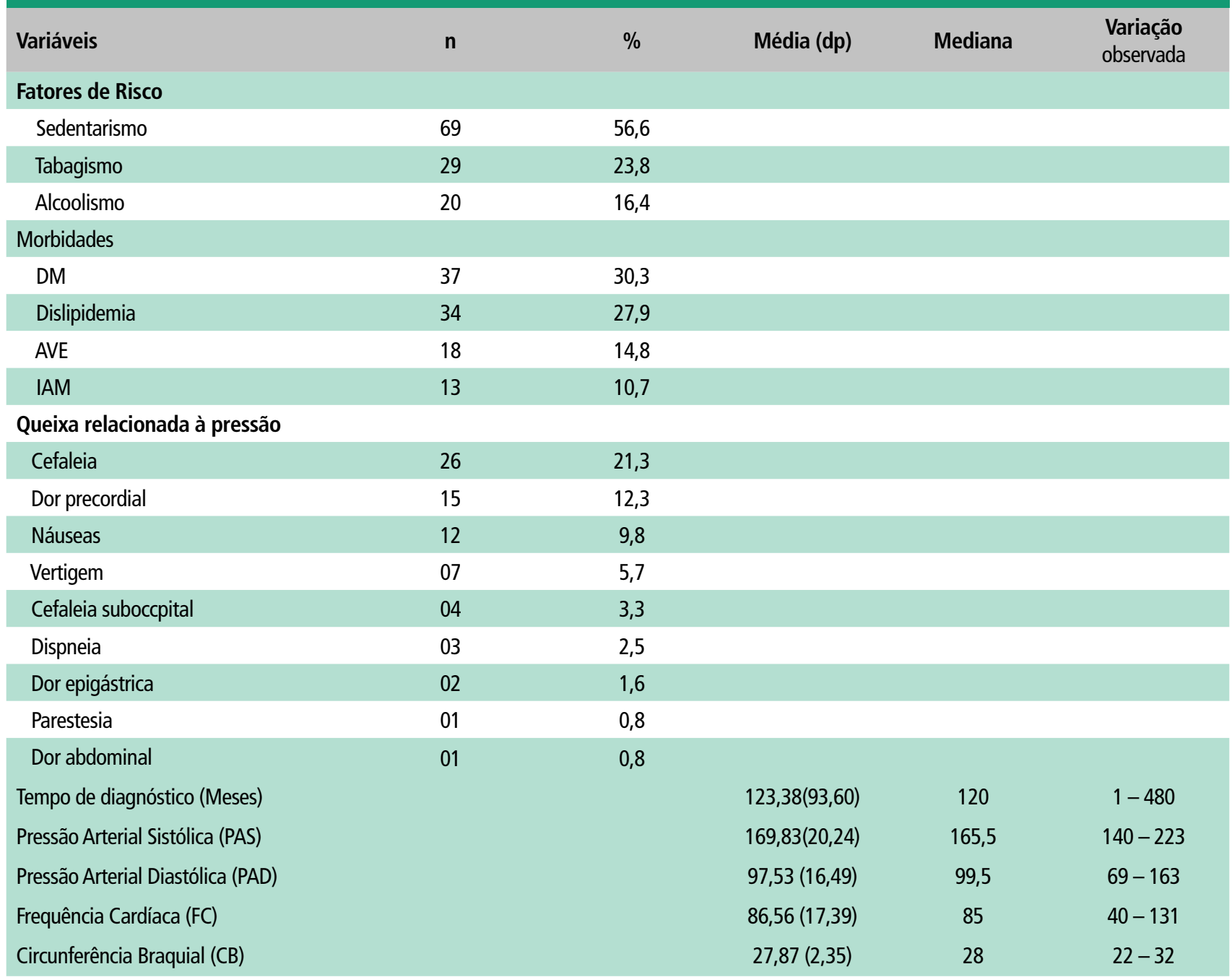

*AVE: Acidente Vascular Encefálico; DM: Diabetes Mellitus; IAM: Infarto Agudo do Miocárdio.

Fonte: Dados da Pesquisa. 
Ao cruzar as variáveis, faixa etária e sexo com os níveis pressóricos e os estágios de hipertensão, constataram-se os dados da tabela 3 .

As queixas atribuídas pelos sujeitos na admissão ao setor são de relevante importância, especialmente para o paciente hipertenso, uma vez que esses pacientes podem apresentar complicações que podem levar a incapacidades ou morte iminente, entre elas:
AVE, ruptura de aneurisma, IAM, entre outros. Assim, cabe ao enfermeiro que atua no setor de triagem o conhecimento dos sinais, sintomas e classificação do estágio da hipertensão para encaminhar o paciente à devida área (azul, verde, amarela ou vermelha) das instituições de pronto atendimento.

Com intuito de demonstrar as associações entre as variáveis, estágios de hiperten- são apresentados e as queixas trazidas pelos hipertensos, elaborou-se a tabela 4.

Considerando-se o teste do Qui-quadrado, observou-se que não houve associação estatisticamente significativa entre dor precordial e PAD; e cefaleia e PAS. Para todas as demais queixas e estadiamentos da PA, apresentaram-se associações estatisticamente significativas $p \geq 0,05$.

Tabela 3: Distribuição dos sujeitos por sexo, faixa etária e estágios da hipertensão arterial. Campina Grande, Paraíba, Brasil, 2017. $(n=122)$

Estagio $1^{\#}$

$\begin{array}{ll}\text { PAS } & \text { PAD } \\ n(\%) & n(\%)\end{array}$

Estagio 2\#

$\begin{array}{llll}\text { PAD } & \text { PAD } & \text { PAD } & \text { PAD } \\ \mathrm{n}(\%) & \mathrm{n}(\%) & \mathrm{n}(\%) & \mathrm{n}(\%)\end{array}$

\section{Sexo}

\begin{tabular}{lcccccc} 
Feminino & $27(22,1)$ & $24(19,7)$ & $32(26,2)$ & $22(18,0)$ & $21(17,2)$ & $15(12,3)$ \\
\hline Masculino & $14(11,5)$ & $14(11,5)$ & $13(10,6)$ & $09(7,4)$ & $15(12,3)$ & $15(12,3)$ \\
\hline Faixa Etária & & & & & 00 & $01(0,8)$ \\
\hline Adulto Jovem & $01(0,8)$ & 00 & $01(0,8)$ & $12(9,8)$ & $17(14,0)$ & $19(15,6)$ \\
\hline Adulto Maduro & $16(13,1)$ & $15(12,3)$ & $16(13,1)$ & $19(15,6)$ & $18(14,8)$ & $10(8,2)$ \\
\hline Idoso & $25(20,5)$ & $23(18,8)$ & $28(22,9)$ & $31(25,4)^{*}$ & $36(29,5)$ & $30(24,6)^{*}$
\end{tabular}

"Estágio 1 - PAS 140-159mmHg/ PAD 90-99 mmHg; Estágio 2 - PAS 160-179mmHg / PAD 100-109mmHg; Estágio 3 - PAS $\geq 180$ mmHg/ PAD $\geq 110 m m H g$;

*O “n” de PAD não soma 122, em virtude dos 23 sujeitos ausentes apresentarem PAD<90, apresentando assim Hipertensão Sistólica Isolada.

Fonte: Dados da Pesquisa.

Tabela 4: Distribuição das queixas de acordo com o estadiamento da hipertensão arterial sistêmica (HAS) no momento da admissão no setor de triagem da UPA 24h. Campina Grande, Paraíba, Brasil, 2017. ( $n=122)$

\begin{tabular}{|c|c|c|c|c|c|c|c|c|}
\hline \multirow{2}{*}{$\begin{array}{l}\text { Queixas relaciona- } \\
\text { das a HAS }\end{array}$} & \multicolumn{2}{|c|}{ Estagio $1^{\#}$} & \multicolumn{2}{|c|}{ Estagio $2^{\#}$} & \multicolumn{2}{|c|}{ Estagio $3^{\#}$} & \multicolumn{2}{|c|}{$p^{*}$} \\
\hline & $\begin{array}{l}\text { PAS } \\
n(\%)\end{array}$ & $\begin{array}{l}\text { PAS } \\
n(\%)\end{array}$ & $\begin{array}{l}\text { PAS } \\
n(\%)\end{array}$ & $\begin{array}{l}\text { PAS } \\
n(\%)\end{array}$ & $\begin{array}{l}\text { PAS } \\
n(\%)\end{array}$ & $\begin{array}{l}\text { PAS } \\
n(\%)\end{array}$ & PAS & PAD \\
\hline Dor Precordial & $04(3,3)$ & $02(1,6)$ & $06(4,9)$ & $03(2,4)$ & $05(4,0)$ & $5(4,0)$ & 0,829 & 0,010 \\
\hline Dor Epigástrica & 00 & 00 & $02(1,6)$ & $01(0,8)$ & 00 & $01(0,8)$ & 0,176 & 0,529 \\
\hline Dispnéia & 00 & 00 & $03(2,4)$ & $01(0,8)$ & 00 & $01(0,8)$ & 0,072 & 0,529 \\
\hline Cefaleia & $03(2,4)$ & $06(4,9)$ & $12(9,8)$ & $06(4,9)$ & $11(9,0)$ & $10(8,2)$ & 0,025 & 0,202 \\
\hline Cefaleia Suboccipital & $01(0,8)$ & $02(1,6)$ & $02(1,6)$ & $01(0,8)$ & $01(0,8)$ & $01(0,8)$ & 0,855 & 0,888 \\
\hline Vertigem & $02(1,6)$ & $03(2,4)$ & $04(3,3)$ & 00 & $01(0,8)$ & $01(0,8)$ & 0,481 & 0,247 \\
\hline
\end{tabular}

* $\mathrm{p}<0,05$ - Teste Qui- quadrado de Pearson

Fonte: Dados da Pesquisa. 


\section{DISCUSSÃO}

Dentre os hipertensos observou-se uma predominância de idosos do sexo feminino9, caracterizando a feminização do envelhecimento. Isto pode ser explicado pelo fato das mulheres viverem mais, estando mais expostas as doenças e consequentemente se fazem mais presentes nos serviços de saúde10.

Observou-se ainda uma maior predominância da faixa etária maiores de 60 anos9. Este achado pode estar relacionado a uma maior proporcionalidade de HAS nos idosos, dificultando assim a sua qualidade de vida em virtude dos sintomas apresentados pelo descontrole da HAS1,11. A idade média dos sujeitos classificava-os como idosos e autodeclarados brancos. Este achado se assemelha aos resultados demonstrados por um estudo realizado na região sudeste do Brasil8, e contrapõe ao estudo ELSA-Brasil que mostrou prevalência de 30,3\% em brancos e 49,3\% em negros 12 .

Evidenciou-se um maior número de indivíduos que moram acompanhados, casados, aposentados, com baixa escolaridade e com renda de aproximadamente um salário mínimo. Este perfil de hipertensos, configura-se como aqueles que abandonam ou não seguem adequadamente o tratamento anti-hipertensivo, ocasionando descontrole pressórico e maior procura pelos serviços de emergência8,13.

Já o levantamento dos dados clínicos, mostrou que as taxas de alcoolismo e tabagismo, considerados fatores de risco para hipertensão, foram baixas para esta amostra. Este achado pode estar relacionado com o perfil de idade dos sujeitos que procuram a UPA 24h apresentando HAS descontrolada.

A Sociedade Brasileira de Cardiologia (SBC)1 defende que o consumo crônico e elevado de bebidas alcoólicas aumenta a PA de forma consistente, e o tabagismo como um fator de risco adicional para doença cardiovascular.

Quando abordados sobre a realização de atividade física, identificou-se que a maioria dos sujeitos não pratica atividade física regularmente. Sabe-se que a prática de atividade física regular apresenta-se como fator importante para o controle dos valores pressóricos 14 .
A SBC constatou que uma pequena parcela dos hipertensos pratica alguma atividade física com regularidade, sendo esse fato relacionado muitas vezes com a falta de ações e indisponibilidade de programas de incentivo1.

Dentre as morbidades, houve uma predominância da DM entre os hipertensos da amostra. Há uma estreita associação entre a diabetes e a HAS, fato ligado à elevação das taxas de sobrepeso e obesidade, o que dobra o risco de DCV. Ambas as doenças, HAS e DM, são consideradas doenças crônicas e de alta prevalência, assintomáticas e que levam a complicações cardiovasculares com consequências incapacitantes aos seus portadores15-16.

Neste estudo, observou-se que os sujeitos apresentavam em seus antecedentes clínicos, algum evento relacionado as complicações decorrentes da hipertensão (DM, dislipidemia, AVE e/ou IAM). Ao serem atendidos em uma UPA24h com HAS descontrolada, acredita-se que estes achados possam estar associados a não adesão ao tratamento farmacológico como também a medidas de controle não farmacológicas, constatada pelo baixo índice de atividade física realizada pelos sujeitos, predispondo os hipertensos a agravos clínicos, com a piora do quadro de saúde em virtude deste descontrole da PA17. Pelo fato de terem passado por estes eventos, esperava-se que estes sujeitos apresentassem níveis pressóricos mais controlados, uma vez que já passaram pela experiência das complicações advindas da HAS.

A maioria dos sujeitos associaram as queixas ao descontrole da HAS, sendo as mais frequentes, relatadas pelos hipertensos e que corroboram com outro estudo10, a cefaleia e dor precordial. A cefaleia é um sintoma comum a diferentes patologias, e motivo de busca por atendimento quando está associada a algum outro sintoma específico, como alterações do nível pressórico e dor precordial, o que se torna motivo de angústia e pressa pela busca dos serviços de saúde. Portanto, constata-se que os hipertensos reconhecem quando os seus valores pressóricos estão alterados, pois buscam a unidade com a queixa relacionada ao seu quadro clinico de descontrole.
Além dessas queixas constataram-se: náusea, dor epigástrica, dispneia, cefaleia suboccipital, vertigem, parestesia e dor abdominal. Esses sintomas podem estar associados muitas vezes a uma lesão em órgão-alvo, caracterizando-se como uma emergência hipertensiva, havendo assim uma estreita relação entre aumento de níveis pressóricos e risco de lesões em órgãos-alvo1,18.

Chamou-nos a atenção para os valores pressóricos mensurados, com uma média de PAS 169,83mmHg e a de PAD 98,49 mmHg. Apesar desses sujeitos apresentarem um leve descontrole, é necessário a classificação de risco adequada, assim como a intervenção imediata, uma vez que há alterações e queixas relacionadas a agravos cardiovasculares, podendo assim evoluir para uma emergência hipertensiva, do tipo crise hipertensiva.

Neste estudo, não se objetivou classificar os riscos que os sujeitos apresentavam, assim como a área para qual eles foram encaminhados, mas cabe destacar a importância do enfermeiro, que lidera uma sala de triagem, apropriar-se de conhecimento para que estes riscos sejam classificados de forma fidedigna e a intervenção seja adequada, minimizando riscos à saúde dos hipertensos triados. Cabe salientar ainda, que estas medidas devem ser realizadas com fidedignidade, e de acordo com as recomendações das VII Diretrizes Brasileiras de Hipertensão1, afim de classificar adequadamente estes hipertensos.

Em relação ao estágio da hipertensão segundo a faixa etária, foi observada uma alta prevalência de idosos 28 (22,9\%) com estágio II (PAS 160-179 mmHg / PAD 100-109 $\mathrm{mmHg}$ ). Outro estudo19 apresentou resultados que diferem dos achados desta pesquisa, ao detectar a faixa etária 41 a 50 anos como a mais prevalente entre os usuários com urgência/emergência hipertensiva atendidos na UPA, com PAS concentrando-se nas faixas de 170-219 mmHg e PAD 120-129 mmHg.

Prezando pelo cuidado em diagnosticar com fidedignidade a pressão arterial dos sujeitos hipertensos que buscaram atendimento e foram inseridos nesta amostra, foi tomada a CB dos sujeitos, a qual apresentou uma variação entre 22-32, mostrando assim a adequação do manguito (tamanho médio) para a afe- 
rição da pressão arterial. Para este estudo foi adquirido um monitor de pressão arterial de braço automático, mais adequado para pesquisa em serviços de urgência, com um manguito que poderia ser acoplado apenas a uma CB de 22-32cm, uma vez que o tamanho do manguito maior que o adequado superestima a pressão, assim como o tamanho menor que o preconizado subestima a pressão20.

Estudo realizado na região nordeste do Brasil21 que buscou identificar a circunferência braquial de pacientes internados em UTI e correlacionar com o tamanho de manguito correspondente, identificou que $65,2 \%$ da amostra estavam utilizando manguitos de tamanhos inadequados às CB. Logo, neste estudo prezou-se por utilizar dados fidedignos e respaldados em literatura científica.

A escolha em adquirir um equipamento para as medidas na pesquisa se deu em relação a ter segurança em estar aferindo de forma adequada as pressões arteriais. Porém a utilização de apenas um tamanho de manguito se tornou uma limitação do estudo, pois restringiu a amostra apenas àqueles que apresentavam a CB padrão,

\section{CONCLUSÃO}

Os pacientes hipertensos não controlados que deram entrada na unidade de pronto atendimento eram a maioria do sexo feminino, idosos, aposentados, casados, de baixa renda e escolaridade, com níveis pressóricos classificados como estágio II de hipertensão. Apresentaram Diabetes Mellitus associado, sedentarismo e cefaleia como queixa principal.

A presença da população hipertensa nos serviços de urgência e emergência, dentre estes, a UPA, serviço que recebe grande parte desta demanda, é um fato preocupante, pois são pacientes que apresentam descontrole dos níveis pressóricos e estão em risco de desencadear complicações decorrentes da morbidade, fatores que estão relacionados a não adesão medicamentosa.

Estes resultados contribuem para o fomento de outras pesquisas, planejamento, execução e avaliação de medidas de promoção e educação em saúde a fim de garantir o fortalecimento da adesão ao tratamento farmacológico e não farmacológico, para promover o controle da pressão arterial.

Espera-se que esses achados subsidiem nos serviços ações de controle da HAS e que os usuários se tornem sujeitos ativos e capazes de promover, intervir e provocar mudanças na vida pessoal e na realidade local em que estão inseridos.

\section{CONFLITO DE INTERESSE}

Os autores declaram que não têm nenhum tipo de interesse econômico, social, pessoal ou de trabalho.

\section{Referências}

1. Sociedade Brasileira de Cardiologia. $7^{\mathrm{a}}$ Diretriz Brasileira de Hipertensão Arterial. Arq Bras Cardiol. 2016;107(3):1-83. doi: http://dx.doi.org/10.5935/abc.20160153 2. Silva EC, Martins MSAS, Guimarães LV, Segri NJ, Lopes MAL, Espinosa MM. Prevalência de hipertensão sistêmica e fatores associados em homens e mulheres em município da Amazônia legal. Rev Bras Epidemiol. 2016;19(1): 38-51. doi: https://doi. org/10.1590/1980-5497201600010004

3. Instituto Brasileiro de Geografia e Estatística (IBGE). Pesquisa Nacional de Saúde 2013: percepção do estado de saúde, estilos de vida e doenças crônicas. Brasil, Grandes Regiões e Unidades da Federação. Rio de Janeiro: IBGE; 2014.

4. Malta DC, Bernal RTI, Andrade SSCA, Silva MMA, Velasquez-Melendez G. Prevalência e fatores associados com hipertensão arterial autorreferida em adultos brasileiros. Rev Saúde Pública. 2017 jun.51(supl.1):1-11. doi:https://doi.org/10.1590/\$15188787.2017051000006

5. Davis $\mathrm{DP}$, Jandrisevits MD, lles S, Weber TR, Gallo LC. Demographic, socioeconomic, and psychological factors related to medication non-adherence among emergency department patients. J Emergency Med. 2012;43(5):773-85. doi: https://doi.org/10.1016/j. jemermed.2009.04.008

6. Brasil. Ministério da Saúde; Secretaria de Atenção à Saúde, Departamento de Atenção Especializada. Manual instrutivo da Rede de Atenção às Urgências e Emergências no Sistema Único de Saúde. $1^{\text {a }}$ ed. Brasilia; 2013.

7. Siqueira ASE, Siqueira-Filho AG, Land MGP.Análise do impacto econômico das doenças cardiovasculares nos últimos cinco anos no Brasil. Arq. Bras. Cardiol. 2017;109(1):39-46. doi: 10.5935/abc.20170068

8. Vancini-Campanharo CR, Oliveira GN, Andrade TFL, Okuno MFP, Lopes MCBT, Batista REA. Hipertensão arterial sistêmica no serviço de emergência: adesão medicamentosa e conhecimento da doença. Rev. Latino-Am. Enfermagem. 2015;23(6):1149-56. doi: https://doi.org/10.1590/0104-1169.0513.2660

9. Gomes IV, Sousa LS, Meneses ASS, Mendes JMS, Almeida XSBA, Almeida TCF. Caracterização dos usuários hipertensos atendidos em unidade de pronto atendimento 24 horas. Nursing. 2018;21(239):2114-8.

10. Siqueira DS, Riegel F, Crossetti MGO, Tavares JP. Perfil de pacientes com crise hipertensiva atendidos em um pronto socorro no sul do Brasil. Rev. Enferm. UFSM. 2015;5(2):22434. doi: $10.5902 / 2179769215316$

11. Oliveira JMM, Nóbrega MML, Medeiros FAL, Medeiros ACT. Characterization of older hipertensive registered in the state of sishiperdia paraiba: allowance for health surveillance J. res.: fundam. care. online. 2015;1(suple.):136-144. doi: http://dx.doi. org/10.9789/2175-5361.2015.v7i5.136-144

12. Chor D, Ribeiro ALP, Canvalho MS, Duncan BB, Lotufo PA, Nobre AA, et al. Prevalen$\mathrm{Ce}$, awareness, treatment and influence of socioeconomic variables on control of high blood pressure: results of the ELSA-Brasil study. POLS One 2015;10(6). doi: https://doi. org/10.1371/journal.pone.0127382

13. Barreto MS, Cremonese IZ, Janeiro V, Matsuda LM, Marcon SS. Prevalence of non-adherence to antihypertensive pharmacotherapy and associated factors. Rev Bras Enferm. 2015;68(1):54-60. doi: http://dx.doi.org/10.1590/0034-7167.2015680109p

14. Bündchen DC, Schenkel IC, Santos RZ, Carvalho T. Exercício físico controla pressão arterial e melhora qualidade de vida. Rev Bras Med Esporte. 2013;19(2):91-5.

15. Radovanovic CAT, Santos LA, Carvalho MDB, Marcon SS. Arterial hypertension and other risk factors associated with cardiovascular diseases among adults. Rev. Latino-Am. Enfermagem. 2014;22(4):547-5. doi: http://dx.doi.org/10.1590/0104-1169.3345.2450 16. López-Jaramillo P, Sánchez RA, Diaz M, Cobos L, Bryce A, Parra-Carillo IZ, et el. Consenso Latino-Americano de hipertensão em pacientes com diabetes tipo 2 e síndrome metabólica. Arq Bras Endocrinol Metab. 2014;58(3):205-25. doi: 10.1590/00042730000003019

17. Lima DBS, Moreira TMM, Borges JWP, Rodrigues MTP.Association between treatment compliance and different types of cardiovascular complications in arterial hypertension patients. Texto contexto-enferm. 2016;25(3):2-9. doi: http://dx.doi.org/10.1590/010407072016000560015

18. Siqueira DS, Riegel F, Crossetti MGO. Tavares JP. Perfil de pacientes com crise hipertensiva atendidos em um pronto socorro no sul do brasil. Rev enferm ufsm. 2015;5(2):22434. doi: $10.5902 / 2179769215316$

19. Jesus PBR, Lopes MHBM, Toti ICC, Silva VFP, Monteiro MI, Lamas JLT. Caracterização e classificação de risco em urgência e emergência hipertensiva. Cogitare Enfermagem. 2016;21(2):1-9. doi: http://dx.doi.org/10.5380/ce.v21i2.43590

20. Destefano RM, Schmitt FRA, Starke S, Helena ETS. Adequação do manguito do esfigmomanômetro às medidas de circunferência braquial em pessoas atendidas na Atenção Primária. Rev. bras. epidemiol. 2017; 20(1):81-90. doi: https://doi.org/10.1590/19805497201700010007

21. Barreto SWS, Oliveira TMF, Gonzaga BO, Arruda CSL, Gouveia Neto JR, Nóbrega NBG. Circunferência braquial e adequação de manguitos em unidade de terapia intensiva adulto. Ciência Saúde. 2017;10(3):121-6.doi: http://dx.doi.org/10.15448/ 1983-652X.2017.3.24565 